\title{
Sharp Front analysis of moisture buffering
}

\author{
Christopher Hall ${ }^{1 *}$, Gloria J. Lo ${ }^{2,3}$, Andrea Hamilton ${ }^{2}$ \\ ${ }^{1}$ School of Engineering, University of Edinburgh \\ 2 Department of Civil and Environmental Engineering, University of Strathclyde \\ ${ }^{3}$ Department of Mechanical and Aerospace Engineering, University of Strathclyde
}

Received: 07 December 2020 / Accepted: 14 July 2021 / Published online: 22 July 2021

(C) The Author(s) 2021. This article is published with open access and licensed under a Creative Commons Attribution 4.0 International License.

\begin{abstract}
Moisture buffering describes the use of high moisture-sorption materials to provide humidity control in interior spaces. Established models of the moisture dynamics of buffering are derived from conventional 1-D Fickian vapour-diffusion equations. We describe an alternative simpler analysis using a 1-D Sharp Front (SF) formulation in which the sorbed water occupies a well-defined zone that extends inwards from the surface. SF modeling is widely used in water transport analyses, but has not previously been applied to a vapour transfer process. This SF analysis emphasizes the (time) ${ }^{1 / 2}$ evolution of the sorption process. It yields the standard expression for the moisture effusivity, several testable scalings and a new definition of the moisture penetration depth. Features of the model are compared with some published experimental data on clay plaster, a widely used buffer material. A new sorption buffer index is a measurable experimental property that describes the water-vapour buffer strength of the material. This index is a composite material property which combines water-vapour sorption capacity and water-vapour permeability, and does not depend on test duration.
\end{abstract}

Keywords: Moisture buffering; Moisture emissivity; Penetration depth; Sharp-Front model; Sorption; Vapour permeability; Sorption buffer index

\section{Introduction}

In buildings subject to episodes of high humidity it is sometimes useful to line interior surfaces with materials that have both high water-vapour permeability and high watervapour sorption capacity. Such materials extract water from humid air, and release it again when the humidity falls. This is the essence of moisture (or humidity) buffering. The idea that materials, whether deliberately introduced or not, may play a consequential part in influencing the humidity of rooms can be traced back at least to Isetti et al. [1], and then to Cunningham [2] and to Koronthályová [3], although the later independent work of Padfield [4] in 1998 was influential.

The topic now has a sizeable research literature. This deals with the moisture dynamics of buffering, material properties, test methods and performance. The established models of buffering dynamics $[5,6]$ are constructed from standard equations for vapour diffusion. These are applied to finite (rather than semi-infinite 'thick' ) 1-D buffer systems with time-dependent (usually step-change) boundary conditions. As a result their solutions involve either rather complicated analytical procedures or fully numerical methods. This is perhaps an impediment to their widescale use, and may also obscure important scaling relations between the controlling variables.
Here we describe an alternative analysis of moisture buffering using a Sharp Front model [7]. SF models often provide mathematically straightforward representations of water transport processes, emphasising the time dependence of mass (or volume) balances. Most SF applications in building physics are of capillary liquid transport, so an SF model of a vapour-transfer process is new. What makes an SF model appropriate for moisture buffering is that most of the water entering the material is immobilised in a compact zone adjacent to the surface. In fact, the strong adsorption of water vapour by typical buffer materials severely retards the rate of vapour diffusion [8]. In consequence most of the mass transfer (both in adsorption and desorption) is confined to a reasonably well-defined sorption zone extending inwards from the surface. We identify the limit of this zone as the location of the Sharp Front. We note that in SF models the advance of the front within a porous material is generally driven by capillary forces acting on liquid water, but is here controlled by the joint effects of vapour diffusion and sorption capacity. Moisture buffering is an example of a highly nonlinear diffusion phenomenon, with the diffusive motion of the front greatly retarded by sorption. This is what makes it appropriate to use an SF formulation.

\footnotetext{
* Corresponding author: Christopher Hall, E-mail: christopher.hall@ed.ac.uk
} 


\section{Buffering dynamics}

\subsection{Water-vapour diffusion}

We consider the dynamics of buffering in the simple slab of Fig. 1.

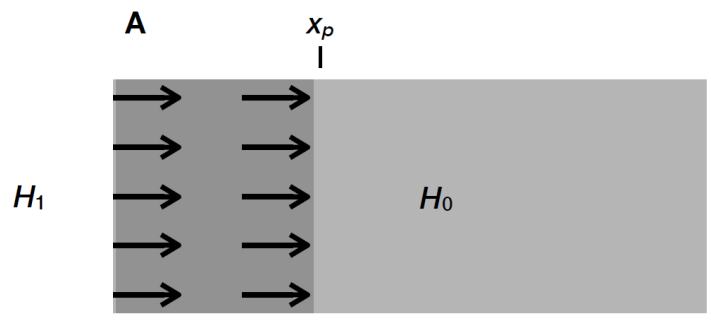

$\mathbf{A}^{\prime}$

Figure 1. A thick slab of uniform material conditioned at humidity $H_{0}$ and subject to a step increase of humidity to $H_{1}$ at the surface $\mathrm{AA}^{\prime}$; $x_{p}$ marks the penetration depth of the advancing sorption front.

The existence of a water-vapour concentration gradient drives water from higher relative humidity to lower. The water-vapour flux can be described by Fick's law of isothermal brownian diffusion

$$
j_{m}=-D_{w} \frac{d c_{w}}{d x}
$$

where $j_{m}$ is the mass flux, $c_{w}$ is the gas-phase water concentration, and $D_{w}$ is a diffusivity with dimension $\mathrm{L}^{2} \mathrm{~T}^{-1}$ (notation here largely follows that of [7]). So long as the water moves through the slab solely by means of molecular diffusion in the gas phase, we can expect $D_{w}$ (at any temperature) to have a constant value independent of $c_{w}$. It is more useful to express $c_{w}$ as a pressure: $c_{w}$ equals the vapour density $\rho_{v}$, and assuming ideal behaviour the water vapour pressure $p_{w}=\rho_{v} R T / M$, where $M$ is the molar mass of water, $R$ the gas constant and $T$ the Kelvin temperature, so that

$$
j_{m}=-\frac{D_{w} M}{R T} \frac{d p_{w}}{d x}=-D_{v} \frac{d p_{w}}{d x} .
$$

In building physics the lumped quantity $D_{v}=\frac{D_{w} M}{R T}$ is called the water vapour permeability [9] and denoted $\delta_{p}$; it is not a permeability in the Darcian sense but rather a quantity proportional to a binary molecular diffusivity. $D_{v}$ has dimension $\mathrm{T}$. The vapour permeability so defined is a property of the slab material expressing the rate at which it transfers water vapour under the action of a gradient of vapour pressure at constant total pressure. It is normally measured directly in a standard cup test [10]. The impeding effect of the porous material is seen by comparing $D_{w}$ with the diffusivity of water vapour in air itself $D_{w 0}$ (usually described as the 'diffusivity in stagnant air' ).

$D_{w 0}$ is a binary gas phase diffusivity but, recognizing that water is usually a minor constituent, the appropriate quantity is the tracer diffusivity, as measured in the dilute limit in which water diffuses in a background of pure air. At $25^{\circ} \mathrm{C} D_{w 0}$ is $2.55 \times 10^{-5} \mathrm{~m}^{2} \mathrm{~s}^{-1}$ [11], and $D_{v 0}$ is $1.85 \times 10^{-10} \mathrm{~s}$. Provided that water is transmitted within the slab solely by molecular diffusion through the gas phase, then the vapour permeability is controlled by the physics of molecular-kinetic diffusion. In particular, diffusivity $D_{w 0}$ varies with absolute temperature $T$ and total pressure $P$ as $T^{n} / P$ where $n$ is around 1.8 [7], so that a similar temperature and pressure dependence is expected for $D_{w}$. Accordingly, $D_{v}$ varies as $T^{n-1} / P$.

\subsection{Water-vapour sorption}

Buffer materials are chosen to sorb water strongly. Information on the sorption property comes from measurements of how the equilibrium water content varies with relative humidity at the temperature of interest. In building physics the sorbed water content is defined in terms either of water content 'mass by volume' $w$ or of water content 'mass by mass' $\theta_{m}$. Here we use the latter. We denote the fractional relative humidity $H=p_{w} / p_{w 0}$, where $p_{w 0}$ is the saturated water vapour pressure. Sorption isotherm datasets $\theta_{m}(H)$ are often fitted to one or other of the many available isotherm functions. The quantity $\xi=$ $\mathrm{d} \theta_{m} / \mathrm{d} H$ is called the moisture (differential) capacity [9]. Alternatively we can define a moisture capacity $\xi_{01}=$ $\Delta \theta_{m} / \Delta H$ from the change in mass $\Delta \theta_{m}$ produced by a prescribed change in relative humidity $\Delta H_{01}=H_{1}-H_{0}$.

\subsection{A Sharp Front description}

We ask how the slab of Fig. 1 responds when the relative humidity at the surface at $\mathrm{AA}^{\prime}$ is increased from $H_{0}$ to $H_{1}$ at time $t=0$. We can apply Eqn 2 to describe the diffusion of water vapour from $\mathrm{AA}^{\prime}$ into the interior of the slab. We recognize that almost all the water entering the material is adsorbed and immobilized, and also that the amount of water adsorbed rises steeply with humidity. (These assumptions are reasonable in the $\mathrm{RH}$ range $\leq 90 \%$ for most practical moisturebuffering materials in which sorbed water is taken up by cellulosic or clay materials). Therefore for $t>0$ the slab develops a zone of higher water content which extends slowly inwards from the surface. If the thickness of this zone is $x_{p}$ (the 'penetration depth') then the cumulative mass of adsorbed water (per unit area of surface) is $i_{m} \approx x_{p} \rho_{b} \Delta \theta_{m}$, where $\Delta \theta_{m}$ is the change in $\theta_{m}$ produced by increasing the humidity by $\Delta H=H_{1}-H_{0}$, and $\rho_{b}$ is the dry bulk density of the slab material. Information about $\theta_{m}(H)$ comes directly from the water-vapour sorption isotherm of the material, so that $\rho_{b} \Delta \theta_{m}=\rho_{b} \xi_{01} \Delta H$. Noting that $H=$ $p_{w} / p_{w 0}$, we have $i_{m} \approx x_{p} \rho_{b} \xi_{01} \Delta p_{w} / p_{w 0}$.

We can write Eqn 2 in the approximate form

$$
\frac{\mathrm{d} i_{m}}{\mathrm{~d} t}=j_{m}=D_{v} \frac{\Delta p_{w}}{x_{p}}
$$

Then eliminating $x_{p}$ gives

$$
i_{m} \frac{\mathrm{d} i_{m}}{\mathrm{~d} t}=\rho_{b} \xi_{01} D_{v} \frac{\left(\Delta p_{w}\right)^{2}}{p_{w 0}}
$$

and integrating with $i_{m}=0$ at $t=0$ we obtain

$$
i_{m}=2^{1 / 2} \Delta p_{w}\left[\frac{\rho_{b} \xi_{01} D_{v}}{p_{w 0}}\right]^{1 / 2} \cdot t^{1 / 2}=2^{1 / 2} \Delta p_{w} \mathrm{~B} \cdot t^{1 / 2}
$$


Here the quantity $\mathrm{B}=\left(\rho_{b} \xi_{01} D_{v} / p_{w 0}\right)^{1 / 2}$, with dimension $\mathrm{L}^{-1} \mathrm{~T}^{3 / 2}$, is the same as the moisture effusivity $b_{m}$ used in the standard analysis of moisture buffering $[5,6]$.

Eqn 5 may be usefully rearranged to give

$$
i_{m}=2^{1 / 2} \Delta H\left(\rho_{b} \xi_{01} D_{v} p_{w 0}\right)^{1 / 2} \cdot t^{1 / 2} .
$$

The quantity $i_{m} /(100 \Delta H)$ is the same, apart from a numerical factor, as the ideal moisture buffer value [MBV] of the standard theory [5], defined for a thick slab.

From Eqn 6 the significant scaling is that, for any time $t, i_{m}$ varies as $[\sim]\left(\rho_{b} \xi_{01} D_{v}\right)^{1 / 2}$, where $\rho_{b}, \xi_{01}, D_{v}$ are material properties. Of these, the moisture capacity $\xi_{01}$ is the quantity with the largest numerical range and the largest impact on the buffer action.

The penetration depth at time $t$ is

$$
x_{p} \approx 2^{1 / 2} \frac{\mathrm{B} p_{w 0}}{\rho_{b} \xi_{01}} \cdot t^{1 / 2}=2^{1 / 2}\left[\frac{D_{v} p_{w 0}}{\rho_{b} \xi_{01}}\right]^{1 / 2} \cdot t^{1.2} .
$$

The main scaling in Eqn 7 is that the penetration depth $x_{p} \sim$ $D_{v}^{1 / 2}$ and $\sim p_{w 0}^{1 / 2}$. Since both $D_{v}$ and $p_{w 0}$ increase with temperature, so also does $x_{p}$. On the other hand $x_{p} \sim$ $1 / \xi_{01}^{1 / 2}$, and so decreases as the moisture sorption capacity increases.

Eqns 6 and 7 taken together show that for a series of materials the quantity $i_{m} / x_{p} \sim \rho_{b} \xi_{01}$ but is independent of $D_{v}$. On the other hand, the quantity $i_{m} x_{p} \sim D_{v}$ but is independent of $\rho_{b} \xi_{01}$. The effect of sorption is to retard the diffusion process, so that the effective water-vapour permeability is $\approx$ $D_{v} /\left(\rho_{b} \xi_{01}\right)$, provided that $\rho_{b} \xi_{01} \gg f \rho_{v 0}$ where $f$ is the volume-fraction porosity of the buffer material and $\rho_{v 0}$ is the saturated water-vapour density at the working temperature.

Eqn 6 can be written as

$$
i_{m}=\mathrm{A} \cdot t^{1 / 2},
$$

where $\mathrm{A}=\Delta H\left(2 \rho_{b} \xi_{01} D_{v} p_{w 0}\right)^{1 / 2}$. We call A (for want of a better term) the sorption buffer index of the material. This quantity $\mathrm{A}$, dimension $\mathrm{ML}^{-2} \mathrm{~T}^{-1 / 2}$, is a directly measurable experimental quantity and is the property that describes the water-vapour buffer strength of the material. The magnitude of $A$ depends both on the moisture capacity and the rate of sorption. Since the moisture capacity $\xi$ is rarely if ever independent of the humidity $H$, the buffer index $\mathrm{A}$ depends on $H$ (or $\Delta H$ ). Arguably, $\mathrm{A}$ is the single most useful quantity to determine experimentally as a measure of buffer performance. It should be noted that the sorption buffer index $\mathrm{A}$ as defined in Eqn 8 is analogous to the sorptivity $S$ in capillary liquid transport models [7, 12]. Unlike the conventional moisture buffer value [5], the index A incorporates the $t^{1 / 2}$ scaling of the sorption process, and therefore is a material property independent of the duration of sorption.

\subsection{Example}

Analysis of published results in dynamic-response tests $[5,13]$ confirms that the mass of water adsorbed following a step change in the imposed humidity generally increases as $t^{1 / 2}$. As an example, Fig. 2 shows data [13] on a clay plaster.

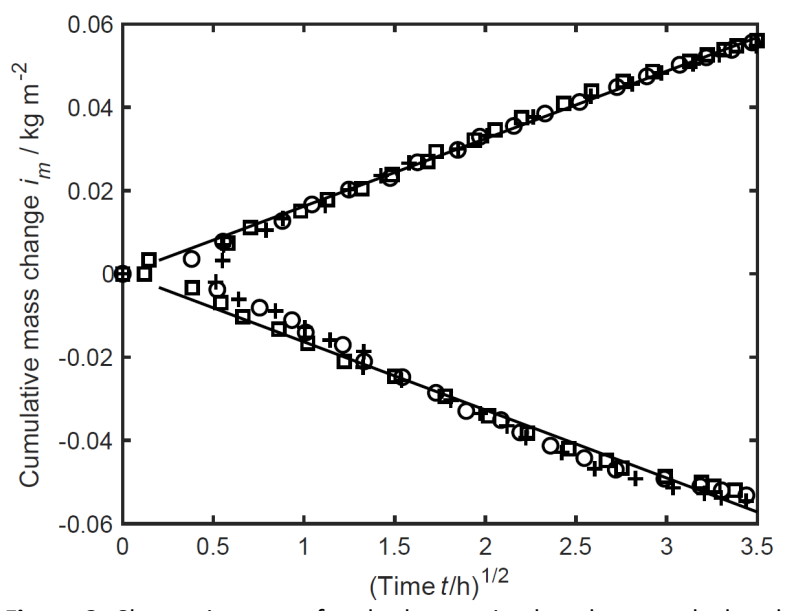

Figure 2. Change in mass of sorbed water in clay plaster, calculated from data of Maskell et al. [13]: three adsorption/desorption cycles, surface condition alternating at $12 \mathrm{~h}$ intervals between $50 \%$ and $75 \% \mathrm{RH}$ at $23^{\circ} \mathrm{C} ; i_{m}$ set to zero at each humidity change; $\mathrm{d} i_{m} /$ $\mathrm{d} t^{1 / 2}=\mathrm{A}= \pm 0.016 \mathrm{~kg} /\left(\mathrm{m}^{2} \mathrm{~h}^{1 / 2}\right)$. First cycle + ; second cycle $\square$; third cycle $\mathrm{o}$.

For this material, and under the test conditions specified, the sorption index is $0.016 \mathrm{~kg} /\left(\mathrm{m}^{2} \mathrm{~h}^{1 / 2}\right)$. In this case, the sorption behaviour is reversible, so that water lost by desorption also increases as $t^{1 / 2}$. The adsorption and desorption rates are similar, and $\mathrm{A}$ has the same value within experimental error. From $\mathrm{A}$ and assuming $D_{v}=2.6 \times 10^{-11} \mathrm{~s}$ at $23^{\circ} \mathrm{C}$ [13], we calculate the position of the penetration front $x_{p}=$ $2 \Delta H D_{v} p_{w 0} / \mathrm{A}=8.4 \mathrm{mmh}^{-1 / 2}$. In other words, the penetration depth $x_{p}=8.4 \mathrm{~mm}$ at $1 \mathrm{~h}$, and $26.4 \mathrm{~mm}$ at $10 \mathrm{~h}$.

\subsection{Comment}

The SF model we describe shares some features with the lumped model of Cunningham [14], which has been adapted for use in moisture buffering [15]. However the SF model makes explicit use of standard parameters $D_{v}$ and $\xi$, and does not depend on a sinusoidal boundary condition. The SF model as briefly presented here does not include surface or interface contact resistances, nor have we discussed multilayer buffers. However SF models readily allow such features to be incorporated [7]. The Nordtest model [5] has a distributed rather than a lumped water content. The analysis supporting the test protocol is based on heat-transfer analogies, and computes the time variation of the watercontent distribution when the material is subject to a squarewave humidity boundary condition. 


\section{Conclusions}

1. A simple Sharp Front model of water vapour sorption has been developed for the main purpose of describing moisture buffering.

2. The SF analysis provides an elementary description of the sorption process, emphasizing the $t^{1 / 2}$ scaling of the cumulative sorption mass changes, the quantities of greatest practical importance.

3. The SF model recovers expressions for cumulative mass change and penetration depth for step changes in humidity which are similar to those of the standard theory.

4. A sorption buffer index is defined to meet the need for a simple experimental measure of the buffer strength of a material.

5. The analysis leads to a number of testable scalings which predict the dependence of the important practical quantities $i_{m}$ and $x_{p}$ on material properties such as $\rho_{b}, D_{v}, \xi_{01}$ and $p_{w 0}$, and hence the temperature variation of $i_{m}$ and $x_{p}$.

The analysis raises several open questions in moisture buffering that require further analysis and experimental investigation.

- Does the cumulative water mass gain vary consistently as $t^{1 / 2}$ as predicted?

- Is the sorption index generally the same for adsorption and desorption?

- What is the true water distribution? There is some NMR imaging data on wood $[16,17]$. However little is known about water distributions in other buffer materials such as clay and lime plasters, often formulated with cellulosic fibres.

- Do buffer materials generally reach sorption equilibrium on the timescales of interest (typically the daily variations in indoor spaces)?

- Is water transport in buffer materials solely by vapour diffusion or is there also film flow (or even, in some cases, true capillary transport)?

\section{Acknowledgments}

GJL thanks the University of Strathclyde, Historic Environment Scotland and BRE for funding from the "Engineering the Future" studentship programme.

\section{Authorship statement (CRediT)}

Christopher Hall: Conceptualization, Formal analysis, Writing - original draft.

Gloria J. Lo: Conceptualization, Investigation, Resources, Writing - review and editing, Funding acquisition.

Andrea Hamilton: Conceptualization, Resources, Writing review and editing, Supervision, Funding acquisition.

\section{References}

[1] C. Isetti, L. Laurenti, A. Ponticiello, Predicting vapour content of the indoor air and latent loads for air-conditioned environments: effect of moisture storage capacity of the walls. Energy and Buildings (1988) 12: 141-148. https://doi.org/10.1016/0378-7788(88)90076-X

[2] M.J. Cunningham, Using hygroscopic damping of relative humidity and vapour pressure fluctuations to measure room ventilation rates. Building and Environment (1994) 29: 501-510. https://doi.org/10.1016/0360-1323(94)90009-4

[3] O. Koronthályová, The effect of the inner surface material on the indoor relative humidity. In International Thermal Energy and Environment Congress Proceedings ITEEC (1997) 97: 557-561.

[4] T. Padfield, The role of absorbent materials in moderating changes of relative humidity. PhD thesis, Technical University of Denmark, 1998.

[5] C. Rode, R.H. Peuhkuri, L.H. Mortensen, K.K. Hansen, B. Time, A. Gustavsen, T. Ojanen, J. Ahonen, K. Svennberg, L-E. Harderup, J. Arfvidsson, Moisture buffering of building materials. Technical University of Denmark, Department of Civil Engineering. BYG Report R-127, 2005.

[6] S. Roels, H. Janssen, A comparison of the Nordtest and Japanese test methods for the moisture buffering performance of building materials. Journal of Building Physics (2006) 30: 137-161. https://doi.org/10.1177/1744259106068101

[7] C. Hall, W.D. Hoff, Water transport in brick, stone and concrete, 2nd edn, Spon Press, London, 2012 https://doi.org/10.1201/b12840

[8] J. Crank, The mathematics of diffusion, 2nd edn, Clarendon Press, Oxford, 1975

[9] ISO 9346:2007. Hygrothermal performance of buildings and building materials - Physical quantities for mass transfer - Vocabulary.

[10] ISO 12572:2016. Hygrothermal performance of building materials and products - Determination of water vapour transmission properties - Cup method.

[11] W.J. Massman, A review of the molecular diffusivities of $\mathrm{H}_{2} \mathrm{O}, \mathrm{CO}_{2}$, $\mathrm{CH}_{4}, \mathrm{CO}, \mathrm{O}_{3}, \mathrm{SO}_{2}, \mathrm{NH}_{3}, \mathrm{~N}_{2} \mathrm{O}, \mathrm{NO}$ and $\mathrm{NO}_{2}$ in air, $\mathrm{O}_{2}$ and $\mathrm{N}_{2}$ near STP. Atmospheric Environment (1998) 32: 1111-1127. https://doi.org/10.1016/S1352-2310(97)00391-9

[12] C. Hall, A. Hamilton, Beyond the sorptivity: definition, measurement and properties of the secondary sorptivity. Journal of Materials in Civil Engineering (2018) 30: 04018049. https://doi.org/10.1061/(ASCE)MT.1943-5533.0002226

[13] D. Maskell, A. Thomson, P. Walker, M. Lemke, Determination of optimal plaster thickness for moisture buffering of indoor air. Building and Environment (2018) 130: 143-150. https://doi.org/10.1016/i.buildenv.2017.11.045

[14] M.J. Cunningham, Effective penetration depth and effective resistance in moisture transfer. Building and Environment (1992) 27: 379-386. https://doi.org/10.1016/0360-1323(92)90037-P

[15] J. Woods, J. Winkler, D. Christensen, Evaluation of the effective moisture penetration depth model for estimating moisture buffering in buildings. NREL/TP-5500-57441, National Renewable Energy Laboratory, Golden, CO, 2013. https://doi.org/10.2172/1219896

[16] S. Hameury, M. Sterley, Magnetic resonance imaging of moisture distribution in Pinus sylvestris L. exposed to daily indoor relative humidity fluctuations. Wood Material Science and Engineering (2006) 1: 116-126. https://doi.org/10.1080/17480270601150578

[17] T. Arends, L. Pel, D. Smeulders, Moisture penetration in oak during sinusoidal humidity fluctuations studied by NMR. Construction and Building Materials (2018) 166: 196-203. https://doi.org/10.1016/j.conbuildmat.2018.01.133 